\title{
MODULAR INEQUALITIES FOR THE MAXIMAL OPERATOR IN VARIABLE LEBESGUE SPACES
}

\author{
DAVID CRUZ-URIBE, OFS, GIOVANNI DI FRATTA, AND ALBERTO FIORENZA
}

\begin{abstract}
A now classical result in the theory of variable Lebesgue spaces due to Lerner 24 is that a modular inequality for the Hardy-Littlewood maximal function in $L^{p(\cdot)}\left(\mathbb{R}^{n}\right)$ holds if and only if the exponent is constant. We generalize this result and give a new and simpler proof. We then find necessary and sufficient conditions for the validity of the weaker modular inequality
\end{abstract}

$$
\int_{\Omega} M f(x)^{p(x)} d x \leq c_{1} \int_{\Omega}|f(x)|^{q(x)} d x+c_{2},
$$

where $c_{1}, c_{2}$ are non-negative constants and $\Omega$ is any measurable subset of $\mathbb{R}^{n}$. As a corollary we get sufficient conditions for the modular inequality

$$
\int_{\Omega}|T f(x)|^{p(x)} d x \leq c_{1} \int_{\Omega}|f(x)|^{q(x)} d x+c_{2},
$$

where $T$ is any operator that is bounded on $L^{p}(\Omega), 1<p<\infty$.

\section{INTRODUCTION}

The variable Lebesgue spaces are a generalization of the classical Lebesgue spaces, where the constant exponent $p$ is replaced by a variable exponent function $p(\cdot)$. They have been studied extensively for the past twenty years, particularly for their applications to PDEs, the calculus of variations [1, 14, 15, but also for their use in a variety of physical and engineering contexts: the modeling of electrorheological fluids [27], the analysis of quasiNewtonian fluids [30], fluid flow in porous media [2], magnetostatics [9] and image reconstruction [4].

Let $\Omega \subset \mathbb{R}^{n}$ be a Lebesgue measurable set, $0<|\Omega| \leq \infty$. Given a measurable exponent function $p(\cdot): \Omega \rightarrow[1, \infty)$, hereafter denoted by $p(\cdot) \in \mathcal{P}(\Omega)$, for any measurable set $E \subset \mathbb{R}^{n},|E \cap \Omega|>0$, we set

$$
p_{-}(E)=\operatorname{ess}_{x \in E \cap \Omega} p(x), \quad p_{+}(E)=\operatorname{ess}_{x \in E \cap \Omega} \sup p(x) .
$$

For brevity, we set $p_{-}=p_{-}(\Omega)$ and $p_{+}=p_{+}(\Omega)$. The space $L^{p(\cdot)}(\Omega)$ is defined as the set of all measurable functions $f$ such that for some $\lambda>0, \rho_{p(\cdot), \Omega}(f / \lambda)<\infty$, where $\rho_{p(\cdot), \Omega}$ is the

1991 Mathematics Subject Classification. 42B25; 46E30; $26 \mathrm{D} 15$.

Key words and phrases. maximal function, variable Lebesgue space, modular inequalities.

The first author is supported by NSF Grant 1362425 and research funds from the Dean of the College of Arts \& Sciences, the University of Alabama.

The second author acknowledges support through the special research program Taming complexity in partial differential systems funded by the Austrian Science Fund (FWF) under grant F65 and of the Vienna Science and Technology Fund (WWTF) through the research project Thermally controlled magnetization dynamics (grant MA14-44). 
modular functional defined by

$$
\rho_{p(\cdot), \Omega}(f)=\int_{\Omega}|f(x)|^{p(x)} d x .
$$

In situations where there is no ambiguity we will simply write $\rho_{p(\cdot)}(f)$ or $\rho(f)$. The space $L^{p(\cdot)}(\Omega)$ is a Banach function space when equipped with the Luxemburg norm

$$
\|f\|_{L^{p(\cdot)}(\Omega)}=\inf \left\{\lambda>0: \rho_{p(\cdot), \Omega}(f / \lambda) \leqslant 1\right\} .
$$

When $p(\cdot)=p$, a constant, then $L^{p(\cdot)}(\Omega)=L^{p}(\Omega)$ and (1.1) reduces to the classical norm on $L^{p}(\Omega)$. For the properties of these spaces, we refer the reader to [14, 15].

Given a function $f \in L_{l o c}^{1}\left(\mathbb{R}^{n}\right)$, the (uncentered) Hardy-Littlewood maximal function $M f$ is defined for $x \in \mathbb{R}^{n}$ by

$$
M f(x)=\sup _{Q \ni x} \frac{1}{|Q|} \int_{Q}|f(y)| d y,
$$

where the supremum is taken over all cubes $Q \subset \mathbb{R}^{n}$ containing $x$ and whose sides are parallel to the coordinate axes. (See [14, 16.) If $f \in L_{l o c}^{1}(\Omega)$, then we define $M f$ by extending $f$ to be identically 0 on $\mathbb{R}^{n} \backslash \Omega$. The following result, proved by Neugebauer and the first and third authors [11, 12], gives a nearly optimal sufficient condition on the exponent $p(\cdot)$ for the maximal operator to satisfy a norm inequality on $L^{p(\cdot)}(\Omega)$.

Theorem 1.1. Given an open set $\Omega \subset \mathbb{R}^{n}$, let $p(\cdot) \in \mathcal{P}(\Omega)$ be such that $1<p_{-} \leq p_{+}<\infty$ and $p(\cdot) \in L H(\Omega)$, i.e., $p(\cdot)$ is log-Hölder continuous both locally and at infinity:

$$
\begin{gathered}
|p(x)-p(y)| \leqslant \frac{C_{0}}{-\log (|x-y|)}, \quad|x-y|<\frac{1}{2}, \quad x, y \in \Omega, \\
\left|p(x)-p_{\infty}\right| \leqslant \frac{C_{\infty}}{\log (e+|x|)}, \quad x \in \Omega .
\end{gathered}
$$

Then $M$ is bounded on $L^{p(\cdot)}(\Omega)$ :

$$
\|M f\|_{L^{p(\cdot)}(\Omega)} \leqslant C\|f\|_{L^{p(\cdot)}(\Omega)} \cdot
$$

In the constant exponent case, Theorem 1.1 reduces to the classical result that the maximal operator is bounded on $L^{p}(\Omega), 1<p<\infty$. In this case, the norm inequality is equivalent to the modular inequality

$$
\int_{\Omega} M f(x)^{p} d x \leq C \int_{\Omega}|f(x)|^{p} d x
$$

Similar modular inequalities hold in the scale of Orlicz spaces: see, for instance, [23]. It is therefore natural to consider the analogous question of modular inequalities for the maximal operator on the variable Lebesgue spaces:

$$
\int_{\Omega} M f(x)^{p(x)} d x \leqslant C \int_{\Omega}|f(x)|^{p(x)} d x .
$$

Since inequality (1.3) implies the norm inequality (1.2), it is clear that stronger hypotheses may be needed on the exponent function $p(\cdot)$ for the modular inequality to hold. The following example from [13] shows that log-Hölder continuity is not sufficient and the modular inequality need not hold even for a smooth exponent function. 
Example 1.2. Let $p(\cdot) \in \mathcal{P}(\mathbb{R})$ be a measurable exponent function which is equal to 2 on the interval $[0,1]$ and equal to 3 on $[2,3]$ (we make no other assumptions on $p(\cdot)$ ). Define the sequence of functions $\left\{f_{k}\right\}_{k \in \mathbb{N}}=\left\{k \chi_{[0,1]}\right\}_{k \in \mathbb{N}}$. Then for any $x \in[2,3]$,

$$
M f_{k}(x) \geqslant \frac{1}{3} \int_{0}^{3}\left|f_{k}(y)\right| d y=\frac{k}{3},
$$

so that

$$
\rho_{p(\cdot), \mathbb{R}}\left(M f_{k}\right) \geqslant \int_{2}^{3}\left(\frac{k}{3}\right)^{3} d x=\frac{k^{3}}{27} .
$$

On the other hand $\rho_{p(\cdot), \mathbb{R}}\left(f_{k}\right)=k^{2}$, so (1.3) cannot hold.

In fact, when $\Omega=\mathbb{R}^{n}$ and $p_{+}<\infty$, Lerner [24] showed that inequality (1.3) never holds unless $p(\cdot)$ is constant.

Theorem 1.3. Let $p(\cdot) \in \mathcal{P}\left(\mathbb{R}^{n}\right), p_{+}<\infty$. Then the modular inequality

$$
\int_{\mathbb{R}^{n}} M f(x)^{p(x)} d x \leqslant C_{p(\cdot), n} \int_{\mathbb{R}^{n}}|f(x)|^{p(x)} d x,
$$

where $C_{p(\cdot), n}$ is a constant depending on $n, p(\cdot)$ but independent of $f$, holds if and only if there is a constant $p>1$ such that $p(\cdot)=p$ almost everywhere.

Remark 1.4. The original proof of Theorem 1.3] in [24] (see also [14, Theorem 3.31]) used the theory of Muckenhoupt $A_{p}$ weights from harmonic analysis. For a simpler proof, see [21] and Corollary 1.22 below.

However, weaker modular inequalities that include an error term are true. These results played a role in the original proofs of Theorem 1.1. For instance, we have the following result [14, Theorem 3.33].

Theorem 1.5. Given $p(\cdot) \in \mathcal{P}\left(\mathbb{R}^{n}\right)$ such that $1<p_{-} \leq p_{+}<\infty$ and $p(\cdot) \in L H\left(\mathbb{R}^{n}\right)$, suppose $f \in L^{p(\cdot)}\left(\mathbb{R}^{n}\right)$ and $\|f\|_{p(\cdot)} \leqslant 1$. Then

$$
\int_{\mathbb{R}^{n}} M f(x)^{p(x)} d x \leqslant C_{p(\cdot), n} \int_{\mathbb{R}^{n}}|f(x)|^{p(x)} d x+C_{p(\cdot), n} \int_{\mathbb{R}^{n}} \frac{d x}{(e+|x|)^{n p-}},
$$

where the constant $C_{p(\cdot), n}$ depends on $n, p(\cdot)$ but is independent of $f$.

The goal of this paper is to give necessary and sufficient conditions for modular inequalities of the form

$$
\int_{\Omega} M f(x)^{p(x)} d x \leqslant c_{1} \int_{\Omega}|f(x)|^{q(x)} d x+c_{2},
$$

to hold for all measurable functions $f$, where $p(\cdot), q(\cdot) \in \mathcal{P}(\Omega)$, and $c_{1}>0, c_{2} \geq 0$ are constants depending on $n, p(\cdot), q(\cdot)$ and $|\Omega|$, but are independent of $f$. We are interested in the weakest possible conditions on the exponent functions $p(\cdot)$ and $q(\cdot)$ for (1.4) to hold. In particular, we want to prove modular inequalities without assuming any smoothness conditions on the exponents.

In this paper we will only consider the case $p(\cdot) \not \equiv 1$. The endpoint case when $p(\cdot) \equiv 1$ is substantially different. If $\Omega$ is bounded and $q_{-}>1$, then (1.4) always holds: this is an immediate consequence of [10, Theorem 1.2]. If $\Omega=\mathbb{R}^{n}$, then (1.4) never holds, since 
$M f$ is never in $L^{1}\left(\mathbb{R}^{n}\right)$ unless $f=0$ a.e. More generally, given any set $\Omega$ with infinite measure, then arguing as in Example 1.7 below, we would have $L^{q(\cdot)}(\Omega) \subset L^{1}(\Omega)$, which is impossible: see [14, Theorem 2.45]. When $q_{-}=1$ the problem of characterizing $q(\cdot)$ is open. Some delicate results in [18, 20] show that this problem depends on how quickly $q(\cdot)$ approaches 1.

Our two main results completely characterize the exponents $p(\cdot)$ and $q(\cdot)$ so that the modular inequality holds. Our characterization depends strongly on whether $\Omega$ has finite or infinite measure; When $\Omega$ has finite measure our result is remarkably simple.

Theorem 1.6. Given a set $\Omega \subseteq \mathbb{R}^{n}, 0<|\Omega|<\infty$, let $p(\cdot), q(\cdot) \in \mathcal{P}(\Omega), p(\cdot) \not \equiv 1$. Then the modular inequality (1.4) holds if and only if $p_{+}(\Omega) \leqslant q_{-}(\Omega)$.

As our second result below shows, the assumption that $|\Omega|<\infty$ is critical in Theorem 1.6. But to motivate this result, we first give the following example.

Example 1.7. If $\Omega \subseteq \mathbb{R}^{n},|\Omega|=\infty$, and if $p(\cdot) \in \mathcal{P}(\Omega), q(\cdot) \in \mathcal{P}(\Omega)$, then the assumption that $p_{+}(\Omega) \leq q_{-}(\Omega)$ is not sufficient for (1.4) to be true. We first consider the case $p_{+}(\Omega)=$ $q_{-}(\Omega)$. Fix an open set $\Omega,|\Omega|=\infty$, and constants $1<p<q<\infty$. Define $p(\cdot) \equiv p$ and

$$
q(x)= \begin{cases}p & \text { if } x \in Q \\ q & \text { if } x \in \Omega \backslash Q\end{cases}
$$

where $Q \subset \Omega$ is a cube. Then $p_{+}(\Omega)=q_{-}(\Omega)$. Suppose (1.4) holds; then we would have

$$
\begin{aligned}
\int_{\Omega}|f(x)|^{p} d x & \leqslant \int_{\Omega} M f(x)^{p} d x \\
& \leqslant c_{1} \int_{Q}|f(x)|^{p} d x+c_{1} \int_{\Omega \backslash Q}|f(x)|^{q} d x+c_{2} .
\end{aligned}
$$

But then, if we let $f:=g \chi_{\Omega \backslash Q}$, we would get the embedding $L^{q}(\Omega \backslash Q) \subset L^{p}(\Omega \backslash Q)$, which does not hold when $p<q$ since $\Omega$ has infinite measure [22, 29].

The case $p_{+}(\Omega)<q_{-}(\Omega)$ is obtained from the same argument by taking $Q=\emptyset$.

The problem in Example 1.7 arises because the exponents $p(\cdot)$ and $q(\cdot)$ behave differently at infinity. To avoid this, we make the following definition.

Definition 1.8. Given a set $\Omega,|\Omega|=\infty$, let $\mathcal{F}_{\Omega}$ denote the collection of subsets of $\Omega$ that havie infinite measure. Given $p(\cdot), q(\cdot) \in \mathcal{P}(\Omega)$, we say that $p(\cdot)$ and $q(\cdot)$ touch at infinity, and denote this by $p(\cdot) \approx q(\cdot)$, if for every $E \in \mathcal{F}_{\Omega}$,

$$
p_{+}(E)=p_{+}(\Omega)=q_{-}(\Omega)=q_{-}(E) .
$$

The exponents in Example 1.7 do not touch at infinity. We consider three additional examples.

Example 1.9. Let $\Omega=\mathbb{R}$.

(1) The exponents $p(x)=2-\left(1+x^{2}\right)^{-1}, q(x)=2+\left(1+x^{2}\right)^{-1}$ touch at infinity.

(2) On the other hand, if we let $\tilde{q}(x)=a+\left(1+x^{2}\right)^{-1}, a>2$, then $p(\cdot)$ and $\tilde{q}(\cdot)$ do not touch at infinity.

(3) Finally, if $p(x) \equiv 2$ and $q(x)=2+\chi_{E}$, where $E$ is any bounded measurable set, then $p(\cdot)$ and $q(\cdot)$ touch at infinity. 
MODULAR INEQUALITIES FOR THE MAXIMAL OPERATOR IN VARIABLE LEBESGUE SPACES 5

We can now state our second main result, characterizing the modular inequality on sets $\Omega$ with infinite measure.

Theorem 1.10. Given a set $\Omega \subseteq \mathbb{R}^{n},|\Omega|=\infty$, let $p(\cdot), q(\cdot) \in \mathcal{P}(\Omega), p(\cdot) \not \equiv 1$. Define $D:=\{x \in \Omega: p(x)<q(x)\} \neq \emptyset$. Then the following are equivalent:

(i) The modular inequality (1.4) holds;

(ii) $p(\cdot) \approx q(\cdot)$ and $L^{q(\cdot)}(\Omega) \hookrightarrow L^{p(\cdot)}(\Omega)$;

(iii) $p(\cdot) \approx q(\cdot)$ and there exists $\lambda>1$ such that

$$
\rho_{r(\cdot), D}(1 / \lambda)=\int_{D} \lambda^{-r(x)} d x<\infty,
$$

where $r(\cdot)$ is the defect exponent defined by $\frac{1}{r(x)}=\frac{1}{p(x)}-\frac{1}{q(x)}$;

(iv) $p(\cdot) \approx q(\cdot)$ and there exists a measurable function $\omega, 0<\omega(\cdot) \leqslant 1$, such that

$$
\rho_{p(\cdot), D}(\omega)=\int_{D} \omega(x)^{p(x)} d x<\infty
$$

and

$$
\left\|\omega(\cdot)^{-\left|p_{+}-p(\cdot)\right|}\right\|_{L^{\infty}(D)} \cdot\left\|\omega(\cdot)^{-\left|q(\cdot)-p_{+}\right|}\right\|_{L^{\infty}(D)}<\infty .
$$

Remark 1.11. There is a close connection between the embedding $L^{q(\cdot)}(\Omega) \hookrightarrow L^{p(\cdot)}(\Omega)$ and condition (1.5): we have that this embedding holds if and only if $p(x) \leq q(x)$ a.e. and (1.5) holds (see [14, Theorem 2.45]). However, (1.5) is independent of $p(\cdot) \approx q(\cdot)$. For one direction, let $\Omega=(2, \infty)$ and define $p(\cdot)$ and $q(\cdot)$ by

$$
\frac{1}{p(x)}=\frac{1}{2}-\frac{1}{x^{2}}, \quad \frac{1}{q(x)}=\frac{1}{p(x)}-\frac{1}{x^{4}} .
$$

Then we have $p(x) \leq q(x)$ and the defect exponent is $r(x)=x^{4}$, so (1.5) holds for any $\lambda>1$. Thus $L^{q(\cdot)}(\Omega) \hookrightarrow L^{p(\cdot)}(\Omega)$. However, $p_{+}(\Omega)=4$ and $q_{-}(\Omega)=2$, so we do not have that $p(\cdot)$ and $q(\cdot)$ touch at infinity.

Conversely, let $\Omega=\left(e^{9}, \infty\right)$ and define $p(\cdot)$ and $q(\cdot)$ by

$$
p(x)=2, \quad q(x)=\frac{2 \log \log (x)}{(\log \log (x)-2)} .
$$

Then $q_{-}(\Omega)=2$ and it decreases to this value as $x \rightarrow \infty$, so $p(\cdot) \approx q(\cdot)$. However, the defect exponent is $r(x)=\log \log (x)$ and the integral in (1.5) is infinite for any value of $\lambda>1$.

Remark 1.12. The condition (1.5) is closely related to the problem of finding sufficient conditions for the maximal operator to be bounded on $L^{p(\cdot)}(\Omega)$ when $\Omega$ is unbounded. Nekvinda [26] showed that the log-Hölder continuity condition at infinity in Theorem 1.1 can be replaced by a weaker integral condition: for some $\lambda>1$,

$$
\int_{D} \lambda^{-r(x)} d x<\infty
$$

where now $r(\cdot)$ is defined by $\frac{1}{r(x)}=\left|\frac{1}{p(x)}-\frac{1}{p_{\infty}}\right|$ and $D=\left\{x \in \Omega: p(x) \neq p_{\infty}\right\}$. For a thorough discussion of this condition and its relationship with (1.5) and the associated embedding theorem, see [14, Section 4.1]. 
Remark 1.13. As a consequence of the assumption that $p(\cdot) \approx q(\cdot)$, we have that for any $R>0$,

$$
p_{+}(\Omega \backslash B(0, R))=p_{+}(\Omega)=q_{-}(\Omega)=q_{-}(\Omega \backslash B(0, R)) .
$$

Therefore, $p(\cdot)$ and $q(\cdot)$ have a common asymptotic value in the sense that

$$
p_{+}(\Omega)=\limsup _{|x| \rightarrow \infty} p(x)=\liminf _{|x| \rightarrow \infty}=q_{-}(\Omega) .
$$

Denote this asymptotic value by $p_{\infty}$; it is a generalization of the value $p_{\infty}$ that occurs in the definition of log-Hölder continuity in Theorem 1.1 or in the Nekvinda condition discussed above. In particular, if $|D|=\infty$, then condition (1.7) is equivalent to

$$
\left\|\omega(\cdot)^{-\left|p_{\infty}-p(\cdot)\right|}\right\|_{L^{\infty}(D)} \cdot\left\|\omega(\cdot)^{-\left|q(\cdot)-p_{\infty}\right|}\right\|_{L^{\infty}(D)}<\infty .
$$

Remark 1.14. It is a consequence of the proof that if the modular inequality (1.4) holds, then $c_{1} \geqslant 1$ : see the proof of the implication $(i) \Rightarrow(i i)$.

In the proof of Theorems 1.6 and 1.10, we use the definition of the maximal operator to prove necessity. In the proof of sufficiency, we only use the fact that the maximal operator is a bounded operator on $L^{p}(\Omega), 1<p<\infty$. Therefore, as an immediate corollary of the proofs we get the following result.

Corollary 1.15. Given a set $\Omega$ and $p(\cdot), q(\cdot) \in \mathcal{P}(\Omega)$, suppose that either $|\Omega|<\infty$ and $p_{+}(\Omega) \leq q_{-}(\Omega)$, or $|\Omega|=\infty, p(\cdot) \approx q(\cdot)$, and (1.5) holds. If $T$ is any operator that is bounded on $L^{p}(\Omega)$ for all $1<p<\infty$, then

$$
\int_{\Omega}|T f(x)|^{p(x)} d x \leq c_{1} \int_{\Omega}|f(x)|^{q(x)} d x+c_{2},
$$

with positive constants $c_{1}, c_{2}$ that depend on $p(\cdot), q(\cdot)$ and $T$ but not on $f$.

The assumption on the operator $T$ is very general and is satisfied by most of the classical operators of harmonic analysis: for example, it holds for Calderón-Zygmund singular integral operators and square functions. In fact, a close examination of the proof shows that we can assume less: given fixed $p(\cdot)$ and $q(\cdot)$, we only require that the operator is bounded on $L^{p_{+}}(\Omega)$. As a consequence, we can prove a modular inequality for the Fourier transform

$$
\hat{f}(\xi)=\int_{\mathbb{R}^{n}} f(x) e^{-2 \pi i x \cdot \xi} d x
$$

on variable Lebesgue spaces, using the Plancherel theorem that $\|\hat{f}\|_{2}=\|f\|_{2}$. The importance of this result follows from the fact that natural generalization of the Hausdorff-Young inequality fails in the variable exponent setting. (See [14, Section 5.6.10] for complete details.)

Corollary 1.16. Given $p(\cdot), q(\cdot) \in \mathcal{P}\left(\mathbb{R}^{n}\right), p_{+}=2$, suppose $p(\cdot) \approx q(\cdot)$, and (1.5) holds. Then

$$
\int_{\mathbb{R}^{n}}|\hat{f}(\xi)|^{p(\xi)} d \xi \leq c_{1} \int_{\mathbb{R}^{n}}|f(x)|^{q(x)} d x+c_{2},
$$

with positive constants $c_{1}, c_{2}$ that depend on $p(\cdot)$ and $q(\cdot)$ but not on $f$. 
Remark 1.17. Modular inequalities for other operators that are bounded on $L^{p}(\Omega)$ have been extensively studied in the setting of Orlicz spaces: see, for example, [5, 6, 7, 8, 17, 23, Modular inequalities in the variable Lebesgue spaces for operators other than the maximal operator have not been studied, though we refer the reader to [19] for a modular interpolation inequality in variable Sobolev spaces.

Remark 1.18. There is a certain parallel between Corollary 1.15 and the theory of Rubio de Francia extrapolation in the scale of variable Lebesgue spaces. Roughly speaking, the theory of extrapolation says that if an operator $T$ is bounded on $L^{p}(w)$, where $1<p<\infty$ and $w$ is any weight in the Muckenhoupt $A_{p}$ class, then $T$ is bounded on $L^{p(\cdot)}$ provided that the maximal operator is bounded on $L^{p(\cdot)}$. (See [14, Section 5.4] for a precise statement of extrapolation.) We can restate Corollary 1.15 as saying that if $T$ is bounded on $L^{p}(\Omega)$, and the maximal operator satisfies a certain modular inequality, then (because Theorems 1.6 and 1.10 given necessary as well as sufficient conditions) $T$ satisfies the same modular inequality (possibly with different constants).

Remark 1.19. It would be of interest to generalize Corollary 1.15 and modular inequalities on Orlicz spaces by considering the analogous question in the scale of Musielak-Orlicz spaces [25]. It would also be interesting to determine if the conditions in Corollary [1.15]are necessary for any other operators to satisfy a modular inequality.

If we consider constant exponent functions $p(\cdot)=p$ and $q(\cdot)=q$, then Theorems 1.6 and 1.10, and Corollary 1.15 have the following corollary.

Corollary 1.20. Given $\Omega \subset \mathbb{R}^{n}$, suppose $|\Omega|<\infty$. If $1<p \leqslant q<\infty$, then the following inequality holds

$$
\int_{\Omega} M f(x)^{p} d x \leqslant c_{1} \int_{\Omega}|f(x)|^{q} d x+c_{2}
$$

for every $f \in L^{q}(\Omega)$ and for some positive constants $c_{1}, c_{2}$ depending on $n, p, q,|\Omega|$, but independent of $f$. If $|\Omega|=\infty$, then inequality (1.8) holds if and only if $1<p=q$.

Moreover, if $T$ is an operator that is bounded on $L^{p}(\Omega), 1<p<\infty$, then these conditions are sufficient for $T$ to satisfy the modular inequality

$$
\int_{\Omega}|T f(x)|^{p} d x \leq c_{1} \int_{\Omega}|f(x)|^{q} d x+c_{2} .
$$

To prove Theorems 1.6 and 1.10, we will first prove the following proposition which establishes a necessary condition which for sets $\Omega$ of finite measure is also sufficient.

Proposition 1.21. Given $p(\cdot), q(\cdot) \in \mathcal{P}(\Omega)$, if the modular inequality (1.4) holds, then

$$
p_{+}(\Omega) \leq q_{-}(\Omega) \text {. }
$$

As a corollary to Proposition 1.21, together with the classical theorem on the boundedness of the maximal operator on $L^{p}(\Omega), 1<p<\infty$ (cf. [28]), we immediately get the following generalization of Theorem 1.3 to arbitrary domains and unbounded exponent functions.

Corollary 1.22. Given an open set $\Omega$ and $p(\cdot) \in \mathcal{P}(\Omega)$, the modular inequality

$$
\int_{\Omega} M f(x)^{p(x)} d x \leqslant c_{1} \int_{\Omega}|f(x)|^{p(x)} d x+c_{2},
$$


with positive constants $c_{1}, c_{2}$ depending on $n, p(\cdot), q(\cdot)$ and $|\Omega|$ but independent of $f$, holds if and only if $p(\cdot)$ equals a constant $p>1$ almost everywhere.

Remark 1.23. Theorem 1.22 does not contradict Theorem 1.5, since in the latter result we need the additional hypothesis that $\|f\|_{L^{p(\cdot)\left(\mathbb{R}^{n}\right)}} \leq 1$.

The remainder of this paper is organized as follows. In Section 2 we first prove Proposition 1.21. In Section 3 we prove Theorem 1.6 and in Section 4 we prove Theorem 1.10.

\section{Proof of Proposition 1.21}

We begin with a definition and a lemma. Given a measurable set $\Omega \subseteq \mathbb{R}^{n},|\Omega|>0$, we denote by $\mathcal{Q}_{\Omega}$ the set of open cubes $Q$ in $\mathbb{R}^{n}$ (whose sides are parallel to the coordinate axes) such that $|\Omega \cap Q|>0$.

Lemma 2.1. Given a set $\Omega \subseteq \mathbb{R}^{n}$, let $p(\cdot) \in \mathcal{P}(\Omega), q(\cdot) \in \mathcal{P}(\Omega)$. Then the following conditions are equivalent:

(i) $p_{+}(Q) \leqslant q_{-}(Q)$ for every $Q \in \mathcal{Q}_{\Omega}$;

(ii) $p_{+}(\Omega) \leqslant q_{-}(\Omega)$.

Proof. The fact that $(i i)$ implies $(i)$ is easy: for any $Q \in \mathcal{Q}_{\Omega}$ we have

$$
p_{+}(Q)=p_{+}(Q \cap \Omega) \leqslant p_{+}(\Omega) \leqslant q_{-}(\Omega) \leqslant q_{-}(Q \cap \Omega)=q_{-}(Q) .
$$

In order to prove that $(i)$ implies $(i i)$, let $\left\{Q_{n}\right\}_{n \in \mathbb{N}}$ be a countable cover of $\Omega$ by elements of $\mathcal{Q}_{\Omega}$. We then have that if $p_{+}(Q) \leq q_{-}(Q)$ for every $Q \in \mathcal{Q}_{\Omega}$, then

$$
p_{+}\left(Q_{m}\right) \leq q_{-}\left(Q_{n}\right) \quad \forall m, n \in \mathbb{N} \text {. }
$$

To see this, note that for every $m, n \in \mathbb{N}$, there exists a cube $Q_{m, n} \in \mathcal{Q}_{\Omega}$ such that $Q_{m} \cup Q_{n} \subseteq$ $Q_{m, n}$. By hypothesis $p_{+}\left(Q_{m, n}\right) \leq q_{-}\left(Q_{m, n}\right)$, so

$$
p_{+}\left(Q_{m}\right) \leqslant p_{+}\left(Q_{m, n}\right) \leqslant q_{-}\left(Q_{m, n}\right) \leqslant q_{-}\left(Q_{n}\right) .
$$

Now, if we first take the supremum over $m \in \mathbb{N}$ and then take the infimum over $n \in \mathbb{N}$, by (2.1) we get $\sup _{m \in \mathbb{N}} p_{+}\left(Q_{m}\right) \leqslant \inf _{n \in \mathbb{N}} q_{-}\left(Q_{n}\right)$. Therefore,

$$
\begin{aligned}
p_{+}(\Omega)=p_{+}\left(\bigcup_{m \in \mathbb{N}} Q_{m}\right) & =\sup _{m \in \mathbb{N}} p_{+}\left(Q_{m}\right) \\
& \leqslant \inf _{n \in \mathbb{N}} q_{-}\left(Q_{n}\right)=q_{-}\left(\bigcup_{n \in \mathbb{N}} Q_{n}\right)=q_{-}(\Omega) .
\end{aligned}
$$

The following argument is inspired by Example 1.2 and is similar to the proof of Theorem 1.3 in [21, Thm. 5.1].

Proof of Proposition 1.21. If (1.9) does not hold, then by Lemma 2.1 there exists a cube $Q \in \mathcal{Q}_{\Omega}$ such that $p_{+}(Q)>q_{-}(Q)$. Let $\alpha, \beta$ be such that

$$
q_{-}(Q)<\alpha<\beta<p_{+}(Q) \text {. }
$$


MODULAR INEQUALITIES FOR THE MAXIMAL OPERATOR IN VARIABLE LEBESGUE SPACES 9

Let $E_{\beta} \subset Q \cap \Omega,\left|E_{\beta}\right|>0$, be such that $p(x) \geq \beta$ for a.e. $x \in E_{\beta}$. Similarly, let $E_{\alpha} \subset Q \cap \Omega$, $\left|E_{\alpha}\right|>0$, be such that $q(x) \leq \alpha$ for a.e. $x \in E_{\alpha}$. Define $f=\lambda \chi_{E_{\alpha}}$, where $\lambda>1$. Then for all $z \in Q$,

$$
M f(z) \geq \frac{1}{|Q|} \int_{Q}|f(y)| d y=\frac{\lambda\left|E_{\alpha}\right|}{|Q|} .
$$

Moreover, if $\lambda>|Q| /\left|E_{\alpha}\right|$, then $\left(\lambda\left|E_{\alpha}\right| /|Q|\right)^{p(x)} \geq\left(\lambda\left|E_{\alpha}\right| /|Q|\right)^{\beta}$ for every $x \in E_{\beta}$. Hence,

$$
\int_{\Omega} M f(x)^{p(x)} d x \geqslant \int_{E_{\beta}}\left(\frac{\lambda\left|E_{\alpha}\right|}{|Q|}\right)^{p(x)} d x \geqslant\left|E_{\beta}\right|\left(\frac{\lambda\left|E_{\alpha}\right|}{|Q|}\right)^{\beta} .
$$

On the other hand,

$$
\int_{\Omega}|f(x)|^{q(x)} d x=\int_{E_{\alpha}} \lambda^{q(x)} d x \leq\left|E_{\alpha}\right| \lambda^{\alpha}
$$

Therefore, if (1.4) holds, then we must have that

$$
\left|E_{\beta}\right|\left(\frac{\lambda\left|E_{\alpha}\right|}{|Q|}\right)^{\beta} \leq c_{1}\left|E_{\alpha}\right| \lambda^{\alpha}+c_{2}
$$

for all $\lambda$ sufficiently large, which is a contradiction since $\alpha<\beta$.

\section{Proof of Theorem 1.6}

By Proposition 1.21 we have that if the modular inequality (1.4) holds, then $p_{+}(\Omega) \leq$ $q_{-}(\Omega)$. Therefore, it remains to show that this condition is sufficient.

Fix a set $\Omega$ and $p(\cdot), q(\cdot) \in \mathcal{P}(\Omega)$ such that $p_{+}(\Omega) \leq q_{-}(\Omega)$, and fix a function $f$. Given a set $E \subseteq \Omega$, we define

$$
I(E)=\int_{E} M f(x)^{p(x)} d x, \quad F(E)=\int_{E}|f(x)|^{p_{+}} d x,
$$

and

$$
D_{1}(M f)=\{x \in \Omega: M f(x)>1\}, \quad D_{1}(f)=\{x \in \Omega:|f(x)|>1\} .
$$

We now estimate as follows:

$$
\int_{\Omega} M f(x) d x=I\left(D_{1}(M f)\right)+I\left(\Omega \backslash D_{1}(M f)\right) .
$$

We immediately have that $I\left(\Omega \backslash D_{1}(M f)\right) \leq|\Omega|$. On the other hand, since $p(\cdot) \not \equiv 1, p_{+}>1$, so the maximal operator is bounded on $L^{p_{+}}(\Omega)$. Hence,

$$
I\left(D_{1}(M f)\right) \leq \int_{D_{1}(M f)} M f(x)^{p_{+}} d x \leq c_{p_{+}, n} \int_{\Omega}|f(x)|^{p_{+}} d x=c_{p_{+}, n} F(\Omega) .
$$

To estimate $F(\Omega)$ we argue similarly: since $p_{+}(\Omega) \leq q_{-}(\Omega)$,

$$
F(\Omega)=F\left(D_{1}(f)\right)+F\left(\Omega \backslash D_{1}(f)\right) \leq \int_{D_{1}(f)}|f(x)|^{q(x)} d x+|\Omega| .
$$

If we combine all of these inequalities, we get

$$
\int_{\Omega} M f(x) d x \leq c_{p_{+}, n} \int_{\Omega}|f(x)|^{q(x)} d x+\left(c_{p_{+}, n}+1\right)|\Omega| .
$$

This completes the proof of sufficiency. 


\section{Proof of Theorem 1.10}

We will prove the following chain of implications:

$$
(i) \Rightarrow(i i) \Rightarrow(i i i) \Rightarrow(i v) \Rightarrow(i) \text {. }
$$

$[(i) \Rightarrow(i i)]$ We first prove that if the modular inequality (1.4) holds, then $L^{q(\cdot)}(\Omega) \hookrightarrow$ $L^{p(\cdot)}(\Omega)$. Since $L^{p(\cdot)}$ is a Banach function space, the embedding $L^{q(\cdot)}(\Omega) \hookrightarrow L^{p(\cdot)}(\Omega)$ is equivalent (cf. [3, Thm. 1.8]) to the set-theoretical inclusion $L^{q(\cdot)}(\Omega) \subseteq L^{p(\cdot)}(\Omega)$. Since $M f(x) \geqslant|f(x)|$ a.e. in $\Omega$, if (1.4) holds, then $\rho_{p(\cdot), \Omega}(f) \leqslant c_{1} \rho_{q(\cdot), \Omega}(f)+c_{2}$. Fix $f \in L^{q(\cdot)}(\Omega)$; then for some $\lambda>0, \rho_{q(\cdot), \Omega}(f / \lambda)<\infty$. Therefore,

$$
\rho_{p(\cdot), \Omega}(f / \lambda) \leqslant c_{1} \rho_{q(\cdot), \Omega}(f / \lambda)+c_{2}<\infty
$$

and so $f \in L^{p(\cdot)}(\Omega)$.

We now prove that if (1.4) holds, then $p(\cdot) \approx q(\cdot)$. Given any measurable set $E \in \mathcal{F}_{\Omega}$ and any measurable function $f: E \subseteq \Omega \rightarrow \mathbb{R}$, (1.4) implies that

$$
\int_{E}|f(x)|^{p(x)} d x \leqslant c_{1} \int_{E}|f(x)|^{q(x)} d x+c_{2},
$$

with $c_{1}, c_{2}>0$ the same constants. Fix $E \in \mathcal{F}_{\Omega}$ and define $f(x)=\lambda \cdot \chi_{B_{\delta} \cap E}(x), 0<\lambda<1$ and $B_{\delta}=B(0, \delta)$. Since $0<\lambda<1$, for $x \in E, \lambda^{p_{+}(E)} \leqslant \lambda^{p(x)}$ and $\lambda^{q(x)} \leqslant \lambda^{q_{-}(E)}$. Therefore, by (4.1),

$$
\begin{aligned}
\left|E \cap B_{\delta}\right| \lambda^{p_{+}(E)} & \leqslant \int_{E \cap B_{\delta}} \lambda^{p(x)} d x \\
& \leqslant c_{1} \int_{E \cap B_{\delta}} \lambda^{q(x)} d x+c_{2} \leqslant c_{1}\left|E \cap B_{\delta}\right| \lambda^{q_{-}(E)}+c_{2} .
\end{aligned}
$$

Since $\left|E \cap B_{\delta}\right| \rightarrow \infty$ as $\delta \rightarrow \infty$, we get that $\lambda^{p_{+}(E)} \leqslant c_{1} \lambda^{q_{-}(E)}+c_{2}\left|E \cap B_{\delta}\right|^{-1}$ for $\delta$ sufficiently large. If we take the limit as $\delta \rightarrow \infty$, we get that if (1.4) holds, then

$$
\lambda^{p_{+}(E)} \leqslant c_{1} \lambda^{q_{-}(E)} \quad \forall 0<\lambda<1 .
$$

Since $p_{+}(E) \leqslant q_{-}(E)$ we must have that $p_{+}(E)=q_{-}(E)$ and $c_{1} \geqslant 1$.

Finally, since by Theorem $1.21, p_{+}(\Omega) \leqslant q_{-}(\Omega)$, and since $p_{+}(E) \leqslant p_{+}(\Omega) \leqslant q_{-}(\Omega) \leqslant$ $q_{-}(E)$, we get that $p(\cdot) \approx q(\cdot)$.

$[(i i) \Rightarrow($ iii $)]$ As noted above, this implication follows from the fact that the embedding $L^{q(\cdot)}(\Omega) \hookrightarrow L^{p(\cdot)}(\Omega)$ is equivalent to assuming $p(x) \leq q(x)$ and (1.5) holds. (See [14, Thm. 2.45].)

$[(i i i) \Rightarrow(i v)]$ We explicitly construct the function $\omega$. Since $p(\cdot) \approx q(\cdot)$, we claim that there exists $\kappa>1$ such that $\left|E_{q(\cdot), \kappa}\right|<\infty$, where $E_{q(\cdot), \kappa}=\{x \in \Omega: q(x)>\kappa\}$. For if not, then for all $\kappa>1,\left|E_{q(\cdot), \kappa}\right|=\infty$. In particular, if we set $\kappa=p_{+}(\Omega)+1$, then $E_{q(\cdot), \kappa} \in \mathcal{F}_{\Omega}$ and $q_{-}\left(E_{q(\cdot), \kappa}\right)>p_{+}(\Omega) \geq p_{+}\left(E_{q(\cdot), \kappa}\right)$, a contradiction.

Fix such a $\kappa$ and define

$$
\omega(x):= \begin{cases}\lambda^{-r(x) / p(x)} & x \in D \backslash E_{q(\cdot), \kappa}, \\ 1 & x \in D \cap E_{q(\cdot), \kappa},\end{cases}
$$


MODULAR INEQUALITIES FOR THE MAXIMAL OPERATOR IN VARIABLE LEBESGUE SPACES11 where $r(\cdot)$ is the defect exponent defined by $\frac{1}{r(x)}=\frac{1}{p(x)}-\frac{1}{q(x)}$. Since $\lambda>1$, we have that $0<\omega(\cdot) \leqslant 1$ and

$$
\begin{aligned}
& \omega(\cdot)^{-\left|p_{+}-p(\cdot)\right|}=\lambda^{\frac{p_{+}-p(\cdot)}{q(\cdot)-p(\cdot)} q(\cdot)} \leqslant \lambda^{q(\cdot)} \leqslant \lambda^{\kappa} \text { on } D \backslash E_{q(\cdot), \kappa}, \\
& \omega(\cdot)^{-\left|q(\cdot)-p_{+}\right|}=\lambda^{\frac{q(\cdot)-p_{+}}{q(\cdot)-p(\cdot)} q(\cdot)} \leqslant \lambda^{q(\cdot)} \leqslant \lambda^{\kappa} \text { on } D \backslash E_{q(\cdot), \kappa .} .
\end{aligned}
$$

Moreover, $\omega(\cdot)^{-\left|p_{+}-p(\cdot)\right|}=\omega(\cdot)^{-\left|q(\cdot)-p_{+}\right|}=1 \leqslant \lambda^{\kappa}$ on the set $D \cap E_{q(\cdot), \kappa}$ and therefore (1.7) holds.

Finally, to prove (1.6) we estimate as follows:

$$
\rho_{p(\cdot), D}(\omega)=\int_{D \backslash E_{q(\cdot), \kappa}} \lambda^{-r(x)} d x+\left|E_{q(\cdot), \kappa}\right| \leqslant \int_{D} \lambda^{-r(x)} d x+\left|E_{q(\cdot), \kappa}\right|<\infty .
$$

$[(i v) \Rightarrow(i)]$ The proof of this implication is similar to the proof of sufficiency in the proof of Theorem 1.6. However, since $|\Omega|=\infty$ we need to introduce $\omega$ and use $\rho_{p(\cdot), D}(\omega)$ in place of $|\Omega|$.

As before, given a measurable function $f$ and a measurable set $E \subseteq \Omega$, define

$$
I(E)=\int_{E} M f(x)^{p(x)} d x, \quad F(E)=\int_{E}|f(x)|^{p_{+}} d x .
$$

Recall that $D=\{x \in \Omega: p(x)<q(x)\}$ and write

$$
\int_{\Omega} M f(x)^{p(x)} d x=I(D)+I(\Omega \backslash D) .
$$

Since $p_{+} \leqslant q_{-}$, we have $p(\cdot)=p_{+}=q_{-}=q(\cdot)$ on $\Omega \backslash D$. Therefore, since $p(\cdot) \not \equiv 1, p_{+}>1$, so the maximal operator is bounded on $L^{p_{+}}(\Omega)$. Hence,

$$
I(\Omega \backslash D)=\int_{\Omega \backslash D} M f(x)^{p_{+}} d x \leqslant c_{p^{+}, n} F(\Omega)
$$

To estimate $I(D)$, define $D_{\omega}(M f)=\{x \in D: M f(x)>\omega(x)\}$ where $\omega$ is the function from our hypothesis $(i v)$. Then

$$
\begin{aligned}
I(D) & =\int_{D \backslash D_{\omega}(M f)} M f(x)^{p(x)} d x+\int_{D_{\omega}(M f)} M f(x)^{p(x)} d x \\
& \leqslant \rho_{p(\cdot), D}(\omega)+\int_{D_{\omega}(M f)}\left(\frac{M f(x)}{\omega(x)}\right)^{p(x)} \omega(x)^{p(x)} d x .
\end{aligned}
$$

Since $M f(\cdot) / \omega(\cdot)>1$ on $D_{\omega}(M f)$,

$$
\begin{aligned}
& \leqslant \rho_{p(\cdot), D}(\omega)+\int_{D_{\omega}\left(M f_{2}\right)}\left(\frac{M f(x)}{\omega(x)}\right)^{p_{+}} \omega(x)^{p(x)} d x \\
& \leqslant \rho_{p(\cdot), D}(\omega)+\left\|\omega^{-\left|p_{+}-p(\cdot)\right|}\right\|_{L^{\infty}(D)} \int_{D}(M f(x))^{p_{+}} d x .
\end{aligned}
$$

Again since $M$ is bounded on $L^{p_{+}}(\Omega)$,

$$
\leqslant \rho_{p(\cdot), D}(\omega)+c_{n, p^{+}} \cdot\left\|\omega^{-\left|p_{+}-p(\cdot)\right|}\right\|_{L^{\infty}(D)} F(\Omega) .
$$


If we combine the above inequalities we get

$$
I(\Omega) \leqslant\left[c_{n, p^{+}}\left(1+\left\|\omega^{-\left|p_{+}-p(\cdot)\right|}\right\|_{L^{\infty}(D)}\right)\right] F(\Omega)+\rho_{p(\cdot), D}(\omega),
$$

so to complete the proof we need to estimate $F(\Omega)=F(D)+F(\Omega \backslash D)$. As before we have $p(\cdot)=p_{+}=q_{-}=q(\cdot)$ on $\Omega \backslash D$, so

$$
F(\Omega \backslash D)=\int_{\Omega \backslash D}|f(x)|^{p_{+}}=\int_{\Omega \backslash D}|f(x)|^{q(x)} d x .
$$

To estimate $F(D)$, let $D_{\omega}(f)=\{x \in D:|f(x)|>\omega(x)\}$. Since $0<\omega \leqslant 1$ and $p_{+} \geqslant p(\cdot)$, we have $\rho_{p_{+}, D}(\omega) \leqslant \rho_{p(\cdot), D}(\omega)$. Therefore,

$$
\begin{aligned}
F(D) & =\int_{D \backslash D_{\omega}(f)}|f(x)|^{p_{+}} d x+\int_{D_{\omega}(f)}|f(x)|^{p_{+}} d x \\
& \leqslant \rho_{p(\cdot), D}(\omega)+\int_{D_{\omega}(f)}\left(\frac{|f(x)|}{\omega(x)}\right)^{p_{+}} \cdot \omega(x)^{p_{+}} d x .
\end{aligned}
$$

Since $|f(\cdot)| / \omega(\cdot)>1$ on $D_{\omega}(f)$

$$
\begin{aligned}
& \leqslant \rho_{p(\cdot), D}(\omega)+\int_{D_{\omega}(f)}\left(\frac{|f(x)|}{\omega(x)}\right)^{q(x)} \cdot \omega(x)^{p_{+}} d x \\
& \leqslant \rho_{p(\cdot), D}(\omega)+\left\|\omega^{-\left|q(\cdot)-p_{+}\right|}\right\|_{L^{\infty}(D)} \int_{D}|f(x)|^{q(x)} d x .
\end{aligned}
$$

If we combine the previous two estimates, we get

$$
\begin{aligned}
F(\Omega) & \leqslant \int_{\Omega \backslash D}|f(x)|^{q(x)} d x+\rho_{p(\cdot), D}(\omega)+\left\|\omega^{-\left|q(\cdot)-p_{+}\right|}\right\|_{L^{\infty}(D)} \int_{D}|f(x)|^{q(x)} d x \\
& \leqslant\left(1+\left\|\omega^{-\left|q(\cdot)-p_{+}\right|}\right\|_{L^{\infty}(D)}\right) \int_{\Omega}|f(x)|^{q(x)} d x+\rho_{p(\cdot), D}(\omega) .
\end{aligned}
$$

Together with inequality (4.2) this gives us the modular inequality (1.4). This completes the proof.

\section{REFERENCES}

[1] E. Acerbi and G. Mingione, Regularity results for stationary electro-rheological fluids, Arch. Ration. Mech. Anal. 164 (2002), no. 3, 213-259.

[2] B. Amaziane, L. Pankratov, and A. Piatnitski, Nonlinear flow through double porosity media in variable exponent Sobolev spaces, Nonlinear Anal. Real World Appl. 10 (2009), no. 4, 2521-2530.

[3] C. Bennett and R. Sharpley, Interpolation of operators, Pure and Applied Mathematics, vol. 129, Academic Press, Inc., Boston, MA, 1988.

[4] P. Blomgren, T. F Chan, P. Mulet, and C.-K. Wong, Total variation image restoration: numerical methods and extensions, in Proceedings of the International Conference on Image Processing, 1997, IEEE, 3 1997, 384-387.

[5] B. Bongioanni, Modular inequalities of maximal operators in Orlicz spaces, Rev. Un. Mat. Argentina 44 (2003), no. 2, 31-47 (2004).

[6] C. Capone and A. Fiorenza, Maximal inequalities in weighted Orlicz spaces, Rend. Accad. Sci. Fis. Mat. Napoli (4) 62 (1995), 213-224 (1996).

[7] M. J. Carro and H. Heinig, Modular inequalities for the Calderón operator, Tohoku Math. J. (2) 52 (2000), no. 1, 31-46. 
[8] M. J. Carro and L. Nikolova, Some extensions of the Marcinkiewicz interpolation theorem in terms of modular inequalities, J. Math. Soc. Japan 55 (2003), no. 2, 385-394.

[9] B. Cekic, A. V. Kalinin, R. A. Mashiyev, and M. Avci, $L^{p(x)}(\Omega)$-estimates of vector fields and some applications to magnetostatics problems, J. Math. Anal. Appl. 389 (2012), no. 2, 838-851.

[10] D. Cruz-Uribe and A. Fiorenza, L $\log L$ results for the maximal operator in variable $L^{p}$ spaces, Trans. Amer. Math. Soc. 361 (2009), no. 5, 2631-2647.

[11] D. Cruz-Uribe, A. Fiorenza, and C. J. Neugebauer, The maximal function on variable $L^{p}$ spaces, Ann. Acad. Sci. Fenn. Math. 28 (2003), no. 1, 223-238.

[12] - Corrections to: "The maximal function on variable L spaces" [Ann. Acad. Sci. Fenn. Math. 28 (2003), no. 1, 223-238; mr1976842], Ann. Acad. Sci. Fenn. Math. 29 (2004), no. 1, 247-249.

[13] D. Cruz-Uribe, The Hardy-Littlewood maximal operator on variable-L ${ }^{p}$ spaces, Seminar of Mathematical Analysis (Malaga/Seville, 2002/2003), Colecc. Abierta, vol. 64, Univ. Sevilla Secr. Publ., Seville, 2003, pp. 147-156.

[14] D. Cruz-Uribe and A. Fiorenza, Variable Lebesgue spaces, Applied and Numerical Harmonic Analysis, Birkhäuser/Springer, Heidelberg, 2013, Foundations and harmonic analysis.

[15] L. Diening, P. Harjulehto, P. Hästö, and M. Růžička, Lebesgue and Sobolev spaces with variable exponents, Lecture Notes in Mathematics, vol. 2017, Springer, Heidelberg, 2011.

[16] J. Duoandikoetxea, Fourier analysis, Graduate Studies in Mathematics, vol. 29, American Mathematical Society, Providence, RI, 2001, Translated and revised from the 1995 Spanish original by D. Cruz-Uribe.

[17] N. Fusco and C. Sbordone, Higher integrability of the gradient of minimizers of functionals with nonstandard growth conditions, Comm. Pure Appl. Math. 43 (1990), no. 5, 673-683.

[18] T. Futamura and Y. Mizuta, Maximal functions for Lebesgue spaces with variable exponent approaching 1, Hiroshima Math. J. 36 (2006), no. 1, 23-28.

[19] F. Giannetti, The modular interpolation inequality in Sobolev spaces with variable exponent attaining the value 1, Math. Inequal. Appl. 14 (2011), no. 3, 509-522.

[20] P. Hästö, The maximal operator in Lebesgue spaces with variable exponent near 1, Math. Nachr. 280 (2007), no. 1-2, 74-82.

[21] M. Izuki, E. Nakai, and Y. Sawano, The Hardy-Littlewood maximal operator on Lebesgue spaces with variable exponent, Harmonic analysis and nonlinear partial differential equations, RIMS Kôkyûroku Bessatsu, B42, Res. Inst. Math. Sci. (RIMS), Kyoto, 2013, pp. 51-94.

[22] V. Kabaila, Inclusion of the space $L^{p}(\mu)$ in $L^{r}(\nu)$, Litovsk. Mat. Sb. 21 (1981), no. 4, $143-148$.

[23] V. Kokilashvili and M. Krbec, Weighted inequalities in Lorentz and Orlicz spaces, World Scientific, 1991.

[24] A. K. Lerner, On modular inequalities in variable $L^{p}$ spaces, Archiv der Math. 85 (2005), no. 6, $538-543$.

[25] J. Musielak. Orlicz Spaces and Modular Spaces, volume 1034 of Lecture Notes in Mathematics. Springer-Verlag, Berlin, 1983.

[26] A. Nekvinda, Hardy-Littlewood maximal operator on $L^{p(x)}\left(\mathbb{R}^{n}\right)$, Math. Inequal. Appl., 7 (2004), no. 2, $255-265$.

[27] M. Růžička, Electrorheological fluids: modeling and mathematical theory, Lecture Notes in Mathematics, vol. 1748, Springer-Verlag, Berlin, 2000.

[28] E. M. Stein, Singular integrals and differentiability properties of functions, vol. 30. Princeton Univ. Press, 1971.

[29] A. Villani, Another note on the inclusion $L^{p}(\mu) \subset L^{q}(\mu)$, Amer. Math. Monthly 92 (1985), no. 7, 485-487.

[30] V. V. Zhikov, Meyer-type estimates for solving the nonlinear Stokes system, Differential Equations 33 (1997), no. 1, 108-115. 
David Cruz-Uribe, OfS, Department of Mathematics, University of Alabama, Tuscaloosa, AL 35487, USA

E-mail address: dcruzuribe@ua.edu

Giovanni Di Fratta, Institute for Analysis and Scientific Computing, TU Wien, Wiedner Hauptstrae 8-10, 1040 Wien, Austria

E-mail address: giovanni.difratta@asc.tuwien.ac.at

Alberto Fiorenza, Dipartimento di Architettura, Università di Napoli, Via Monteoliveto, 3, I-80134 Napoli, Italy, and Istituto Per le Applicazioni del Calcolo "Mauro Picone", sezione di Napoli, Consiglio Nazionale delle Ricerche, via Pietro Castellino, 111, I-80131 NAPOLI, ITALY

E-mail address: fiorenza@unina.it 